International Journal of Electrical and Power Engineering 4 (2): 85-89, 2010

ISSN: 1990-7958

(C) Medwell Journals, 2010

\title{
Electric Power Demand Forecasting of KAVAL Cities
}

\author{
${ }^{1}$ A.K. Bhardwaj, ${ }^{2}$ R.C. Bansal, ${ }^{3}$ R.K. Saket and ${ }^{1}$ A.K. Srivastava \\ ${ }^{1}$ Shepherd School of Engineering and Technology, Sam Higganbottom Institute of Agriculture, \\ Technology and Sciences-Deemed University (Formerly AAI-DU), Allahabad, India \\ ${ }^{2}$ School of Information Technology and Electrical Engineering, \\ University of Queensland, St. Lucia, Q1d-4072, Australia \\ ${ }^{3}$ Institute of Technology, Banaras Hindu University, Electerical Engineering, Varanasi, India
}

\begin{abstract}
Power load forecasting is having its own importance in a bulk interconnected combined generation, transmission and distribution power system. Accurately load forecasting is important to establish operational plans for power stations and their generation units, implementation of improved plant scheduling. It further provides a reliable and credible resource that ensures stable and economical operation of the power. The crucial issue of managing the demand is of a great importance in formulating the future development policy for the whole country. The case studies analyses the requirement of electricity with respect to the future population for the major forms of energy in the KAVAL cities of Uttar Pradesh state in India. A model consisting of significant key energy indicators have been used for the estimation. Model wherever required refined in the second stage to remove the effect of auto-correlation. The accuracy of the model has been checked using standard statistical techniques and validated against the past data by testing for expost forecast accuracy. The study identifies the urgent need for special attention in evolving effective energy policies to alleviate an energy famine in the near future.
\end{abstract}

Key words: Energy forecasting, energy modeling, power demand, expost forecast, KAVAL cities, India

\section{INTRODUCTION}

As power is one of the fundamental infrastructure input for the development, its prospects and availability sets significant constraints on the socio-economic growth of the state. A care full long-term power plan is imperative for the development of power sector. This need assumes more importance in the state of Uttar Pradesh where the demand for electrical energy is growing rapidly.

Since electricity is one of the necessities in the ordinary business of life and a major driving force for economic growth and development. The non-storable nature of electricity means that the supply of electricity must be always available to satisfy the growing demand. Since the commission of power plants and the transmission may take between 5-7 years and power purchase from another source is limited, it is imperative that the power development plan must be well conceived. Inevitably, a reliable medium and long run load forecasts are prerequisites for a well-conceived power development plan. An under forecasted load leads to an under expanded power system which leads to the black out of power system. On the other hand, an over forecasted load leads to an over expanded power system. In this case; the unnecessary costs are passed on to the power consumers through a higher power tariff.

Load forecasting has always been important for planning and operational decision conducted by utility companies. However, with the deregulation of the energy industries, load forecasting is even more important. With supply and demand fluctuating and the changes of weather conditions and energy prices increasing by a factor of 10 or more during peak situations, load forecasting is vitally important for utilities.

There are several factors that affect electricity demand. The key factors are electricity price, number of electricity appliances, income, temperature and consumer load pattern that differ by regions and consumer groups (Bansal and Pandey, 2005; Yee et al., 2009). A reliable load forecast methodology must correctly gauge the effects of the key factors on electricity demand. The electricity demand which includes public lighting, will be forecasted by customer groups (NIDA Consulting Center, 2006). On the other hand, utilization hours for electric power

Corresponding Author: A.K. Bhardwaj, Shepherd School of Engineering and Technology,

Sam Higganbottom Institute of Agriculture,

Technology and Sciences-Deemed University (Formerly AAI-DU), Allahabad, India 
generation equipments has continued to drop since 2000 and will continue to decline in 2008 , according to predictions of the Power Grid Corporation, De-rated output operation or even shutting down units of power generation during low demand periods not only increases energy consumption but also affects equipment life span and is overall uneconomical. Uttar Pradesh is a state located in the northern part of India with a population of over 19 million people and an area of 93,933 mile $^{2}$ $\left(243,286 \mathrm{~km}^{2}\right)$.

It is India's most populous state, as well as the world's most populous sub-national entity. The demand of power is increasing day by day in the daily life. So forecasting of power is very important for the future. The state was having deficit in power supply of $-12.6 \%$ of total demand in June 2007 while in June 2008, it was having the deficit of $-13.7 \%$.

In another way, transmission and distribution losses in $2002-03$ was $36.64 \%$ and in $2005-06$ it was $37.17 \%$ of the total power supply in the state. As per the 2001 Indian Census (Govternment of India, 2001), population and percentage with respect to the total population with temperature range are shown in Table 1.

This study has studied the power forecast in 2023 corresponding to the city temperatures with respect to the population in the cities (Bhardwaj and Bansal, 2010). Few researches are conducted with respect to the population (Sharma et al., 2002), as well as temperature of the particular areas (Barakat and Al-Rashed, 1992) individually while few are written with cosideration of both factors (Bhardwaj et al., 2009; Bhardwaj and Bansal, 2010).

\section{Calculation of population and load survey}

Population calculation: Growth rate is essential value to find the population in a specified year based on a particular time.

The time frame can be defined between the standard base year and final year with respect to the time span of 9 months. Following formula can be suggested for growth rate calculation (Bhardwaj et al., 2009).

\begin{tabular}{llll}
\multicolumn{4}{l}{ Table 1: Population and maximum } \\
\hline $\begin{array}{l}\text { Name of } \\
\text { the city }\end{array}$ & $\begin{array}{l}\text { Population in } 1991 \\
\text { and \% w.r.t. } \\
\text { total population }\end{array}$ & $\begin{array}{l}\text { Population in } 2001 \\
\text { and \% w.r.t. } \\
\text { total population }\end{array}$ & $\begin{array}{l}\text { Max. } \\
\text { temp. in } \\
\text { summer }\left({ }^{\circ} \mathrm{C}\right)\end{array}$ \\
\hline Kanpur & $\begin{array}{l}22,16,973 \\
(2.46 \%)\end{array}$ & $\begin{array}{l}26,26,918 \\
(2.49 \%)\end{array}$ & 47.5 \\
Allahabad & $8,56,897$ & $10,15,348$ & \\
& $(2.95 \%)$ & $(2.98 \%)$ & 47.0 \\
Varanasi & $9,43,525$ & $11,17,994$ & \\
& $(1.90 \%)$ & $(1.90 \%)$ & 46.0 \\
Agra & $11,11,086$ & $13,16,177$ & \\
& $(2.08 \%)$ & $(2.17 \%)$ & 40.0 \\
Lucknow & $19,13,166$ & $22,66,933$ & \\
& $(2.09 \%)$ & $(2.22 \%)$ & 45.0 \\
\hline
\end{tabular}

$$
\mathrm{N}=\mathrm{B}\left[\{(100+\mathrm{G}) / 100\}^{\mathrm{T}}-1\right]
$$

Where:

$$
\begin{aligned}
\mathrm{N}= & \text { Net increase in population between } 2001 \text { and } \\
& 1991 \\
\mathrm{~B}= & \text { Base year value of } 1991 \\
\mathrm{G}= & \text { Growth rate }(1.28 \%) \\
\mathrm{T}= & \text { Total period between } 2001 \text { and } 1991
\end{aligned}
$$

Since the correct data of 2008 is not available, so in this particular calculation, we will take 2001 value as base year value and time span between 2001 and 2008 i.e., 9.34. Population in the cities using the relation = $\mathrm{B}\{(100+\mathrm{G}) / 100\}^{\mathrm{T}}$ are shown in Table 2 . In all the cases of estimate of population, we have taken the base year value of 2001 and time periods between 2001 and the required year.

Load survey: Load survey was carried out in the month of June 2008 from the transmission Sub-station which are having in coming from the Grid.

The loads are taken from $132 \mathrm{kV}$ sides of the transformers. Following results are shown in Fig.1. As per the trend the net power demand for the KAVAL cities individually are shown in Table 3.

Per capita demand for the year 2001, 2008 and 2023 with respect to the load and population are shown in Table 4.

Table 2: Estimated population

\begin{tabular}{llll}
\hline & Years & & \\
Name of & - & & \\
the city & 2008 & 2015 & 2023 \\
\hline Kanpur & $29,58,270$ & $33,30,994$ & $38,15,150$ \\
Allahabad & $11,43,421$ & $12,87,485$ & $14,74,619$ \\
Varanasi & $12,59,014$ & $14,17,642$ & $16,23,489$ \\
Agra & $14,82,195$ & $16,68,943$ & $19,11,279$ \\
Lucknow & $25,52,877$ & $28,74,524$ & $32,91,914$ \\
\hline
\end{tabular}

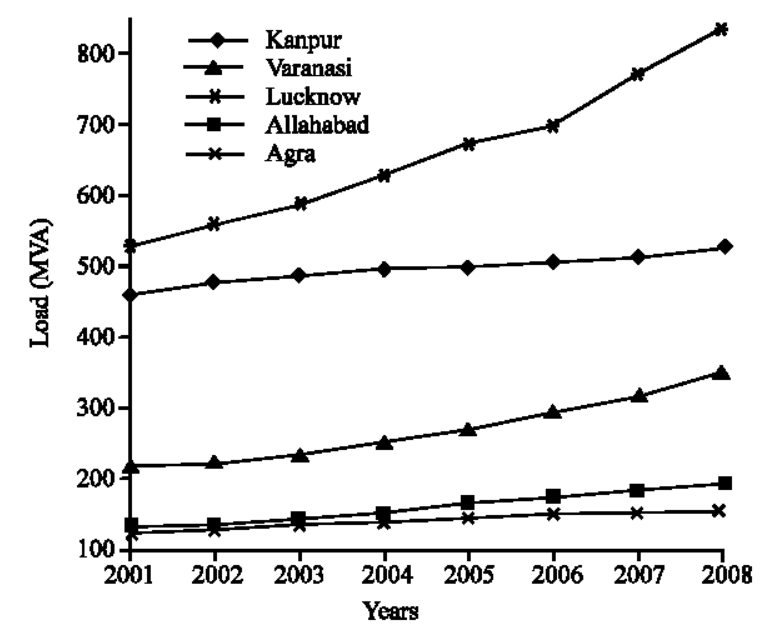

Fig. 1: Total load 2001-2008 
Int. J. Elec. Power Eng., 4 (2): 85-89, 2010

Table 3: Net forecast power demand (MVA)

\begin{tabular}{llcr}
\hline & Years & & \\
Name of & - & & \\
the city & 2015 & 634.0 & 2023 \\
\hline Kanpur & 585.0 & 392.0 & 666.0 \\
Allahabad & 291.5 & 780.0 & 467.5 \\
Varanasi & 552.0 & 233.0 & 957.0 \\
Agra & 198.0 & 1768.0 & 256.5 \\
Lucknow & 1279.0 & & 2143.0 \\
\hline
\end{tabular}

Table 4: Per-capita demand

\begin{tabular}{|c|c|c|c|}
\hline \multirow{2}{*}{$\begin{array}{l}\text { Name of } \\
\text { the city }\end{array}$} & \multicolumn{3}{|l|}{ Years } \\
\hline & 2001 & 2008 & 2023 \\
\hline Kanpur & $1.74 \times 10^{4}$ & $1.76 \times 10^{4}$ & $1.74 \times 10^{4}$ \\
\hline Allahabad & $1.29 \times 10^{4}$ & $1.69 \times 10^{4}$ & $3.17 \times 10^{4}$ \\
\hline Varanasi & $1.95 \times 10^{4}$ & $2.77 \times 10^{-4}$ & $5.89 \times 10^{4}$ \\
\hline Agra & $9.75 \times 10^{5}$ & $1.04 \times 10^{4}$ & $1.34 \times 10^{4}$ \\
\hline Lucknow & $2.31 \times 10^{4}$ & $3.26 \times 10^{4}$ & $6.51 \times 10^{4}$ \\
\hline
\end{tabular}

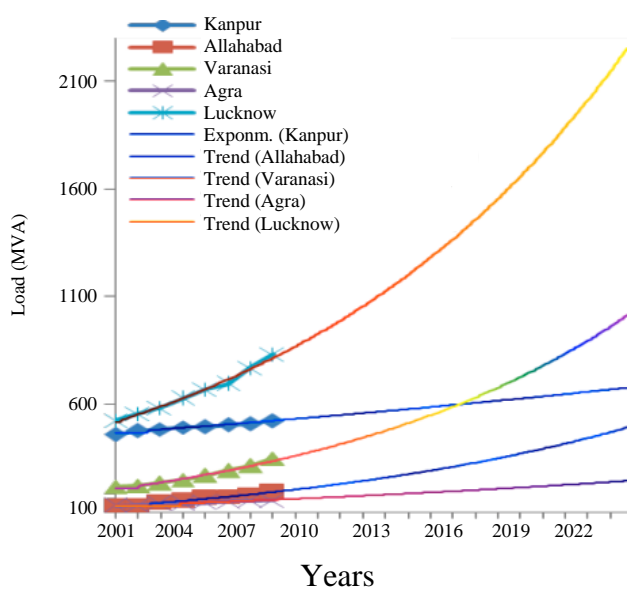

Fig. 2: Forecast trend for 2023

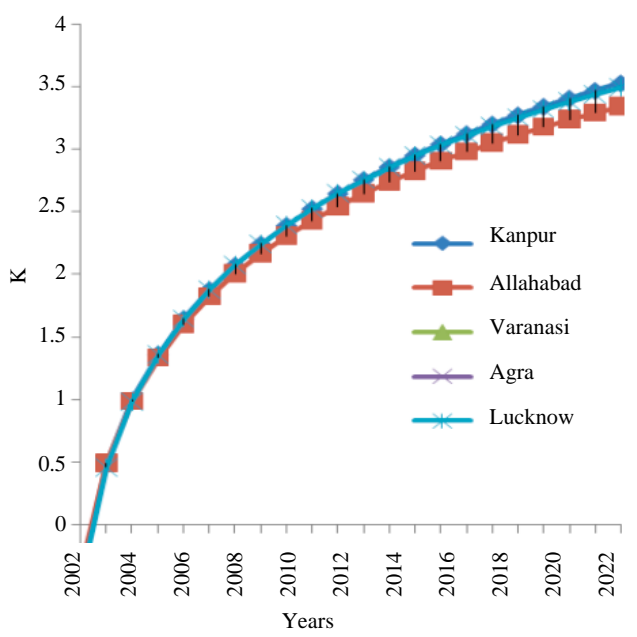

Fig. 3: The values of $\mathrm{K}$ for KAVAL cities

The model: The final modified model proposed (Bhardwaj et al., 2009) for consumption of electricity is of the following functional form:

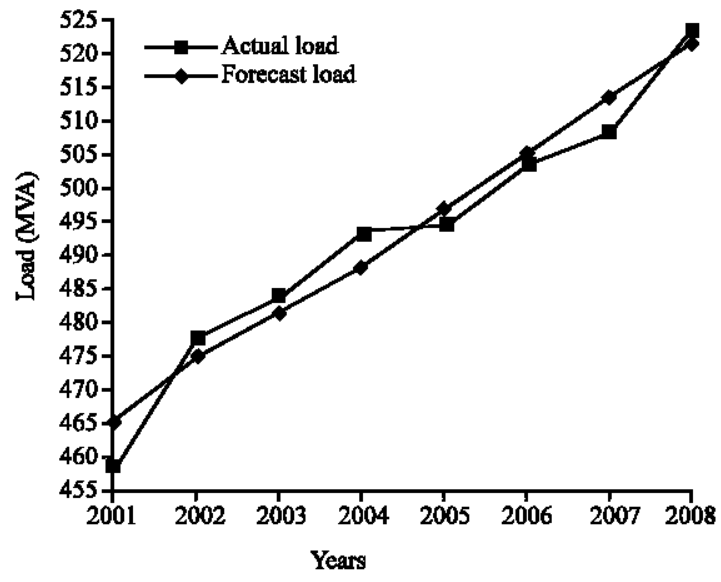

Fig. 4: Kanpur load comparison

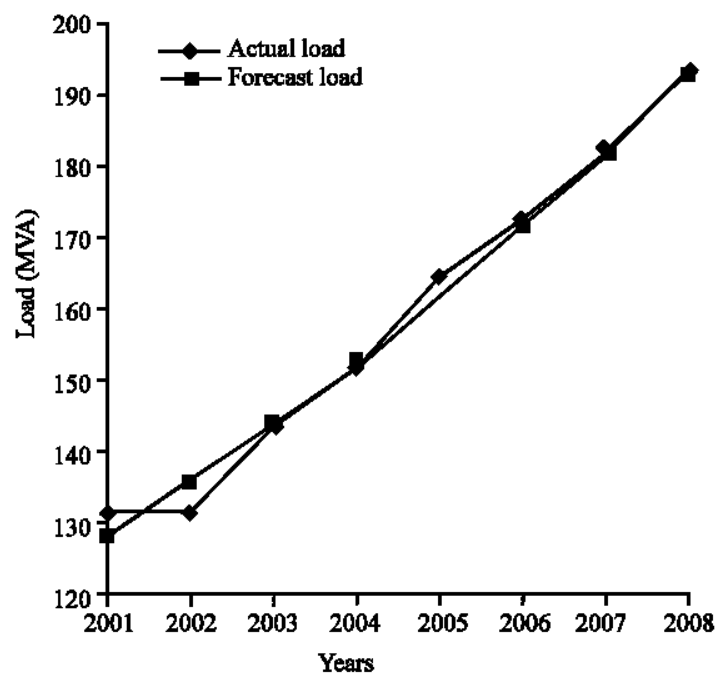

Fig. 5: Allahabad load comparison

$$
\begin{gathered}
\ln (\mathrm{Ei} / \mathrm{Pi})=\mathrm{K} \ln (\mathrm{Eb} / \mathrm{Pb})-3.48881 \\
\ln (\mathrm{T} / 10)-10.99549 \ln \{1 /(\mathrm{Yi}-\mathrm{Yb})\}
\end{gathered}
$$

Where $\mathrm{i}$ and $\mathrm{b}$ represents the values of corresponding subscripts to the ith year and that of the base year (2001) (Fig. 2) and E, P, Y and T are electricity demand, population, year and absolute temperature of the city in ${ }^{\circ} \mathrm{C}$, respectively. The value of constant $\mathrm{K}$ can be obtained from Fig 3. Figure 3 shows that the value of constant $\mathrm{K}$ except Allahabad city is almost same and it will be varying from -0.40002 to +3.52951 while value of Allahabad city will very between -0.35203 to 3.34472 .

Analysis of model accuracy: In order to ascertain the accuracy of the above model, the plots comparing the actual consumption with the model estimation have been verified. The plots shows acceptable level of accuracy of 
Int. J.Elec. Power Eng., 4 (2): 85-89, 2010
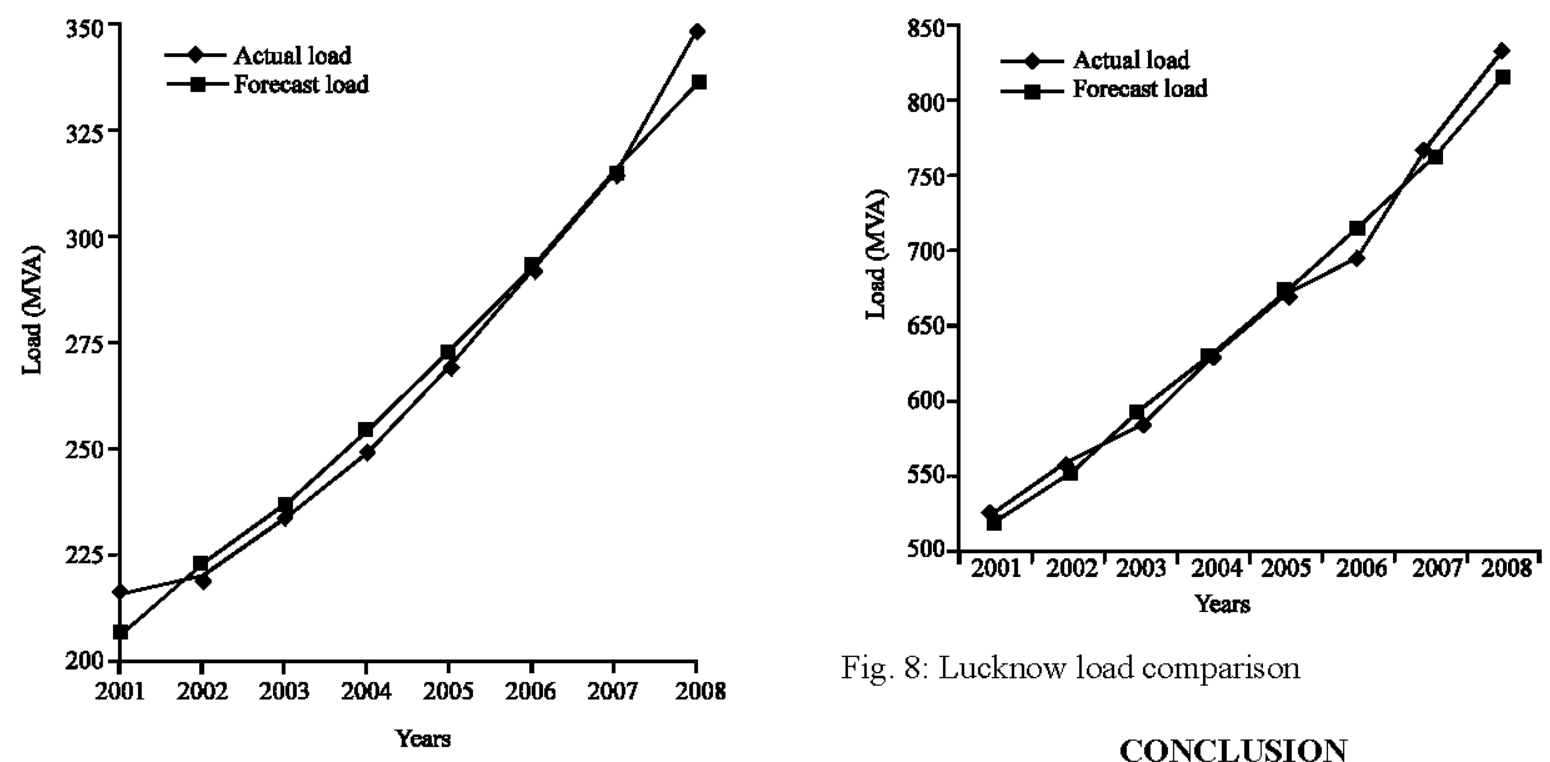

Fig. 8: Lucknow load comparison

Fig. 6: Varanasi load comparison

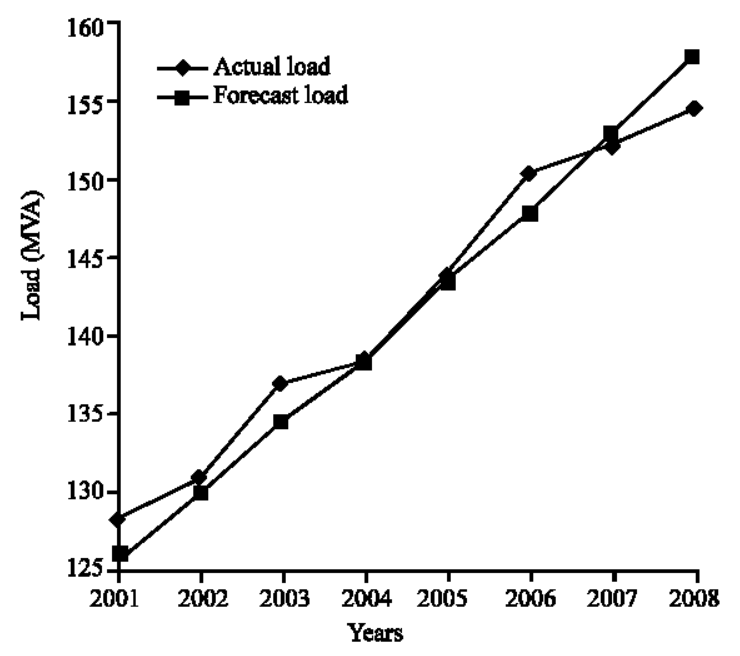

Fig. 7: Agra load comparison

the model. The analysis of actual load with forecast load comparison gives result that the Kanpur has an average error (Fig. 4) of $+0.07 \%$ (Bhardwaj, 2010).

Similarly analysis of actual load with forecast load comparison gives result (Fig. 5) that the Allahabad has an average error of $-0.04 \%$ (Bhardwaj et al., 2009).

The Fig. 6 of actual load with forecast load comparison gives result that the Varanasi has an average error of $-0.21 \%$ (Barakat and Al-Rashed, 1992).

According to the analysis of actual load with forecast load (Fig. 7) comparison Agra has an average error of $-0.44 \%$ (Bhardwaj et al., 2009) and Lucknow has an average error of $+0.08 \%$ (Fig. 8) (Bhardwaj and Bansal, 2010).

\section{CONCLUSION}

Long term planning is of a vital significance for the growth of power sector. The study emphasizes the urgent need for an effective long term planning for generation expansion to meet the state's rapidly growing demand. The results project the enormous capacity requirement for realizing the power demand by the year 2023 . The analysis gives result that the Kanpur has almost same per capita demand as compare with maximum temperature while Varanasi has a increase of per capita demand approximate 3 times when compare with a constant maximum temperature. It is also clear that the Allahabad has more per capita demand as compare with Agra because maximum temperature of Allahabad is $6-7^{\circ} \mathrm{C}$ higher than the Agra.

Analysis shows that the Lucknow has an increase of per capita demand approximate 3 times when compare with a constant maximum temperature which we have taken i.e., $45^{\circ} \mathrm{C}$. However, as per the Global warming report temperature will rise up to $3^{\circ} \mathrm{C}$ till the year 2050 . The study indicates various options available now a day to meet the power demand by 2023 i.e., increases additional generation capacity, reducing the present level of transmission and distribution losses, etc.

A long term plan based on the per capita demand can meet the requirement of power in the future. In this study, a model has been verified which explains the demand of electrical energy till the year 2023 .

From the results, the possibility of improving the forecasting accuracy of conventional approaches by using this proposed method is verified.

The model has been refined and validated to ensure consistency. Error of the forecast model has also been checked and that is found to be in the range with an 
average error of $-0.11 \%$. The model has been employed for forecasting the future energy requirement until 2023. The policy implications of the forecast results have been critically analyzed. The results identify the basis on which electricity energy planning can be carried out and emphasize the need for evolving effective strategies.

\section{ACKNOWLEDGEMENT}

The researchers gratefully acknowledge the advice and help rendered by Shri B.P. Sharma (Retired) Dy. Director, Central Electricity Authority, New Delhi, India in carrying out this research.

\section{REFERENCES}

Bansal, R.C. and J.C. Pandey, 2005. Load forecasting using artificial intelligence techniques: A literature survey. Int. J. Comp. Appl. Technol., 22: 109-119.

Barakat, E.H. and S.A. Al-Rashed, 1992. Long range peak demand forecasting under conditions of high growth. IEEE Trans. Power Syst., 7: 1483-1 486.
Bhardwaj, A.K., A.K. Srivastava and R.C. Bansal, 2009. Electric power demand forecasting: A case study of Agra and Allahabad cities. Electrical India Magazine, 49: 40-44.

Bhardwaj, A.K. and R.C. Bansal, 2010. Electric power demand forecasting: A case study of Lucknow city. Subseed Science Magazine, April 2010.

Bhardwaj, A.K., 2010. Electric power demand forecasting: A case study of kanpur city. Proceeding of the National Seminar on Utilisation and Reliability of Power System: An Indian Scenario, Jan. 16, SIET Allahabad (India), pp: 84-84.

NIDA Consulting Center, 2006. Thailand Long-Term Load Forecasts. Chapter 8, National Institute of Development Administration, Thailand, pp: 109-124.

Sharma, D.P., P.S.C. Nair and R. Balasubramanian, 2002. Demand for commercial energy in the state of Kerala, India: An econometric analysis with medium-range projection. Energy Policy, 30: 781-791.

Yee, S., R.C. Bansal, A.K. Bhardwaj and A.K. Srivastava, 2009. Electricity market price forecasting using vector support machines. Electric Power Comp. Syst., 37: 495-516. 